\title{
Efecto de un programa de "exergames" en el equilibrio y la movilidad funcional de personas mayores: un estudio piloto
}

\author{
Fernando Labra Gómez ${ }^{\mathrm{a}, \mathrm{c}}$, Sandra Mahecha Matsudo ${ }^{\mathrm{b}, \mathrm{c}}$ \\ a Kinesiólogo. fernandolabra@live.com.mx iD https://orcid.org/0000-0003-3692-4996 \\ b MD. Ph. D.; Post Doctorado en Ciencias del envejecimiento. Esp. Medicina Deportiva. \\ ' Facultad de Ciencias, Universidad Mayor. Santiago, Chile \\ DOI 10.22517/25395203.24081
}

\section{Resumen}

Introducción: Las personas mayores experimentan una menor capacidad para mantener y restablecer el equilibrio durante las actividades físicas. Durante la última década los videojuegos controlados con movimientos de todo el cuerpo, también conocidos como "Exergames", han ganado popularidad como herramientas para mejorar el equilibrio y la movilidad funcional. El software KineLabs $3 D$ es una serie de minijuegos que utiliza la plataforma Kinect para la rehabilitación de pacientes con secuelas de accidente vascular encefálico y para el entrenamiento de personas mayores. A nuestro conocimiento no hay investigaciones que utilizen este Exergame.

Objetivo: Analizar el efecto de un programa de ejercicios del Exergame KineLabs $3 D$ en el equilibrio y la movilidad funcional en personas institucionalizadas y autovalentes entre $\operatorname{los} 65$ y los 85 años.

Materiales y métodos: Se realiza un estudio piloto, cuantitativo, pre test-post test de un solo grupo de cinco personas que completaron un programa de 24 sesiones, tres veces por semana. Las pruebas clínicas escala de balance de Berg, prueba de alcance funcional, estación de apoyo unipodal y Short Physical Performance Battery fueron utilizadas antes y después del desarrollo del programa para medir el equilibrio y la movilidad funcional.

Resultados: Las personas mayores que participaron en el programa mostraron una diferencia significativa en todas las pruebas clínicas (escala de balance de Berg $\mathrm{p}=0,0422$, prueba de alcance funcional $\mathrm{p}=0,0431$, estación de apoyo unipodal $\mathrm{p}=0,0422$ y Short Physical Performance Battery $\mathrm{p}=0,0384$; $\mathrm{p}<0,05)$

Conclusiones:Un programa de ocho semanas de ejercicios de Exergames en personas autovalentes institucionalizadas mejoró el equilibrio y movilidad funcional.

Palabras clave: Video juegos, realidad virtual, envejecimiento, envejecido, caídas, balance postural.
A pilot study of effects of Exergames program on balance and functional mobility in older adults

\begin{abstract}
:
Introduction: Older people experience a lower capacity in maintaining and resetting balance throughout the physical performance. During the last decade, video games that imply controlled full body-actions that are known as 'Exergames' have gained popularity as tools to improve balance and functional mobility. KineLabs 3D software is a mini-games series that employs the Kinect platform to rehabilitate patients with brain stroke damage and for senior people training. As far as we know, there are no research studies that use this Exergame currently.
\end{abstract}

Objective: To analyze the effect of an Exergame KineLabs 3D training program regarding the balance and functional mobility in self-sufficient and institutionalized people between 65 and 85 years old.

Materials and methods: This quantitative pilot study was carried out in a group of 5 people that attended a 24-session program three times per week. To measure stance and functional mobility, clinical testing such as Berg's balance test, Functional reach test, Unipedal stance test, and Short physical performance battery test were used before and after the intervention.

Results: Older people that participated in the program showed a significant difference within all clinical tests made. The results were: Berg Balance Scale $\mathrm{p}=0,0422$, Functional Reach Test $\mathrm{p}=0,0431$, Unipedal Stance Test $\mathrm{p}=0,0422$ and Short Physical Performance Battery $\mathrm{p}=0,0384$; $\mathrm{p}$ less than 0,05 .

Conclusion: The implementation of an eight-week Exergames program enhanced the stance/balance and functional mobility in the participants.

Key words: Video games, virtual reality, aging, postural balance 


\section{Introducción}

El deterioro del control postural se encuentra entre las principales causas de caídas ${ }_{(1)}$ en las personas mayores, puesto que esta población experimenta una menor capacidad para mantener y restablecer el equilibrio durante las actividades físicas ${ }_{(1)}$ Se ha encontrado que las intervenciones con ejercicios físicos que entrenan las estrategias de equilibrio son $50 \%$ más efectivas para prevenir caídas, probablemente debido a que promueven un mejor equilibrio, una mayor movilidad y un menor tiempo de reacción ${ }_{(2)}$.

Durante la última década, los videojuegos controlados con movimientos de todo el cuerpo, también conocidos como "Exergames" (EG), han ganado popularidad como herramientas para mejorar el equilibrio y la movilidad funcional ${ }_{(1)}$. Podemos definir el equilibrio como el proceso por el cual se controla el centro de masa del cuerpo respecto a la base de sustentación, ya sea estática o dinámica ${ }_{(3)}$; mientras que movilidad funcional es la capacidad fisiológica para moverse de forma independiente y segura en una variedad de entornos con el fin de realizar actividades o tareas funcionales y participar en actividades de la vida diaria en el hogar, el trabajo y la comunidad ${ }_{(4)}$.

Dentro de los EG destaca la tecnología capturadora de movimiento "Kinect" (Microsoft Corp., Redmond, WA) debido a que es una de las plataformas más populares ${ }_{(5)}$. Esta reconoce los movimientos corporales mientras que el jugador es representado en el videojuego como un "avatar virtual", el cual, gracias a la adquisición de datos en forma instantánea, reproduce los desplazamientos y acciones del usuario. La sencillez en el manejo de la plataforma hace que el proceso sea comprendido fácilmente por la persona mayor, aún si carece de experiencia previa en el dominio de videojuegos ${ }_{(6)}$. En este sentido, la evidencia actual respalda el uso terapéutico de los EG como estrategia para mejorar el equilibrio ${ }_{(5)}$. Además, es percibida positivamente por las personas mayores ${ }_{(7)}$, no obstante, no es concluyente si esta tecnología es superior a la terapia convencional ${ }_{(8)(9)}$.

Probablemente, debido a la heterogeneidad de las variables y de los instrumentos de medición ${ }_{(10)}$, Donath et al. ${ }_{(9)}$ concluyeron en su meta análisis que aun cuando es un poco menos efectivo que la terapia convencional (consistente en un programa de ejercicios de equilibrio tradicional), el entrenamiento basado en EG es un método aceptable para mejorar el equilibrio y los movimientos funcionales de las personas mayores ${ }_{(9)}$. Además, las caracteristicas tecnológicas de la plataforma Kinect han permitido que desarrolladores independientes creen EG con aplicaciones terapeuticas (6) (11) (12) (13) (14) (2) (15) , siendo más seguros y efectivos que los EG comerciales ${ }_{(16)}$.

La Organización Mundial de la Salud (OMS) recomienda que las personas mayores realicen ejercicios para mejorar el equilibrio y disminuir la prevalencia de caídas ${ }_{(17)}$, pero la adherencia a programas que incluya este tipo de actividades suele ser deficiente ${ }_{(1)}$. Además, la falta de tiempo, motivación, aburrimiento, miedo a caerse, y factores como inconveniencia, inaccesibilidad, seguridad y costo ${ }_{(2)}$, son otras barreras que afectan la realización de ejercicio entre la población mayor.

En este sentido, la posibilidad de usar los EG como una estrategia para superar las barreras que limitan la actividad física de las personas mayores, ha motivado a los investigadores a realizar estudios piloto de entrenamiento no supervisado, tanto en el hogar como en instituciones. Al respecto, Van Diest et al. ${ }_{(1)}$ y Garcia et al. ${ }_{(2)}$ utilizaron EG terapéuticos de desarrollo independiente para sus investigaciones. Ambos estudios, con resultados similares, señalan que el entrenamiento sin supervisión es factible, seguro y efectivo (2) (1)

Por tanto, el objetivo de la presente investigación analizar el efecto de un programa de ocho semanas de ejercicios del Exergame KineLabs $3 D$ en el equilibrio y la movilidad funcional en personas de 65 a 85 años, autovalentes e institucionalizadas. El software KineLabs $3 D$ es una serie de minijuegos que utiliza la plataforma Kinect para la rehabilitación de pacientes con secuelas de accidente vascular encefálico y para el entrenamiento de personas mayores. Con su uso, los pacientes pueden entrenar en el hogar, instituciones y hospitales ${ }_{(18)}$.

\section{Materiales y métodos}

Se llevó a cabo un estudio piloto cuantitativo, pre test-post test de un solo grupo, un subtipo de diseño intra-sujeto perteneciente a los estudios cuasi experimentales ${ }_{(19)}$. La población de estudio corresponde al establecimiento de larga estadía para adultos mayores (ELEAM) "Fundación Casa Betania", donde habitan 15 personas, 5 de las cuales participaron en el programa piloto. Esta fundación sin fines de lucro atiende a personas de ambos sexos, de 65 años en adelante, dependientes leves a severos, autovalentes con riesgo y en riesgo de dependencia, en situación vulnerable de la comuna de Santiago. Se realizó un muestreo no probabilístico por conveniencia y se incluyeron 5 sujetos (3 femeninos y 2 masculinos), a quienes se les hizo entrega de un consentimiento informado que fue revisado y firmado por cada uno de los participantes.

Los criterios de inclusión fueron: tener entre 65 a 85 años, ser autovalente y no presentar señales de deterioro cognitivo. Se excluyeron aquellas personas que presentaban algún tipo de condición de salud inestable incompatible con las actividades realizadas en el programa como mareos y vértigo, enfermedades crónicas no controladas, ceguera, daltonismo, adherencia menor al $70 \%$ de las sesiones y analfabetismo. Según datos de auto reporte ninguno de los sujetos tenía experiencia previa en Exergames. El estudio fue aprobado por el Comité de Ética Científico de la Universidad Mayor, número de protocolo 198 con fecha del 27 de junio del 2019.

Los participantes realizaron cuatro pruebas clínicas: escala de balance de Berg, prueba de alcance funcional, estación de apoyo unipodal y Short Physical Performance Battery. La escala de balance de Berg determina objetivamente la capacidad para mantener el equilibrio, tanto estático como 
dinámico, mediante una serie de 14 tareas evaluadas con un puntaje de 0 a 4 , siendo 56 el máximo ${ }_{(20)}$. Se ha determinado que personas mayores con puntajes menores a 45 tienen un gran riesgo de caída ${ }_{(21)}$.

La prueba de alcance funcional es una prueba desarrollada por Duncan et al. ${ }_{(22)}$ como un medio rápido para medir equilibrio dinámico en adultos mayores (22), los participantes son instruidos a ubicarse en postura bípeda con los pies separados a la distancia de los hombros y con un brazo (mano en un puño) elevado a 90 grados de flexión. Sin mover los pies, los sujetos avanzan tan lejos como puedan mientras mantienen su equilibrio, se mide la distancia alcanzada y se compara con valores normativos ${ }_{(22)}$. Una distancia menor a $18,5 \mathrm{~cm}$ en personas mayores puede ser indicador de riesgo de caída ${ }_{(23)}$.

La estación de apoyo unipodal es una prueba simple para cuantificar el equilibrio estático ${ }_{(24)}$, en ella los participantes deben situarse con los brazos cruzados apoyados sobre los hombros, a la orden del evaluador deben mantener una extremidad inferior en una posición de $90^{\circ}$ de flexión en cadera y rodilla. Se mide el tiempo en que la persona es capaz de mantener esta posición, la prueba se realiza tres veces en cada lado registrando el mejor tiempo obtenido. Se considera como equilibrio normal, aquella persona que es capaz de mantener la posición descrita por más de 5 segundos ${ }_{(25)}$.

La Short Physical Performance Battery (SPPB) consiste en una serie de pruebas diseñadas para medir la movilidad funcional y el rendimiento físico del adulto mayor. El puntaje se calcula a través de tres componentes: la habilidad de estar en posición bípeda por 10 segundos con los pies en tres posiciones (paralelos, semi tándem y tándem); tiempo en recorrer caminando 4 metros y tiempo en ponerse de pie 5 veces desde una silla ${ }_{(26)}$. El tiempo medido para cada una de las tres pruebas se convierte en valores de escala ordinales de 0 a 4. Las puntuaciones de las tres áreas se suman para un rango total de 0 que equivale a menor rendimiento y a 12 que indica mayor rendimiento ${ }_{(22)}$. Un puntaje igual o menor a 6 en personas mayores está asociado con un mayor riesgo de caída

(27) ${ }^{\circ}$

Luego de las mediciones iniciales, los participantes iniciaron las sesiones de ejercicios virtuales con el Software KineLabs $3 D$. Este consiste en una serie de minijuegos con niveles de dificultad personalizables que utilizan la plataforma Kinect (18) El software no solo cumple con los objetivos primarios de un juego (ganar, avanzar etapas, aumentar puntaje) sino que también los secundarios (precisión y calidad en la ejecución de los movimientos que controla el avatar). Esto permite categorizar este software como un EG terapéutico ${ }_{(28)}$. Los minijuegos utilizados fueron: Good View Hunting $(\mathrm{GVH})$ y Cockroach Invasion (CI). En GVH los participantes deben mantener limpia una ventana, para ello deben tomar elementos de aseo (paños, esponjas, etc.) y limpiar con los brazos las distintas suciedades que aparecen como hollín, manchas de agua, suciedad de pájaro y rayados de dibujos infantiles. En
CI los participantes deben pisar cucarachas, las que presentan distinto tamaño y cantidad de vida. Dependiendo de cuan alto se levante el pie será la potencia necesaria para eliminarlas. En ambos minijuegos, conforme avanza el tiempo, los objetivos se hacen más difíciles de lograr, por lo que el participante debe aumentar la frecuencia, amplitud de movimiento y rapidez. El puntaje final se asigna en función de la velocidad y precisión con la que se desempeñan, mientras que el tiempo de duración de las partidas, sin posibilidad de pausarlas o detenerlas, fue de 6 y 8 minutos para GVH y CI respectivamente.

El entrenamiento se llevó a cabo en la sala multiuso del establecimiento, acondicionada para la realización de la actividad. Los participantes debían asistir con ropa cómoda y calzado adecuado (zapatilla, zapato cerrado, zapato con tacón de altura fisiológica). Los ejercicios se realizaron de forma individual durante 30 minutos, tiempo aproximado que tomaba programar y completar dos partidas de GVH y dos de CI. La frecuencia de la actividad fue de 3 veces por semana, para un total de 24 sesiones.

Si bien la sesión fue supervisada por los investigadores, no se brindó asistencia física. La labor de los investigadores se limitó a programar los dispositivos al inicio de la sesión, ofrecer a los participantes instrucciones verbales y, en la medida de lo necesario, recordar el objetivo del juego. Conforme avanzaban las sesiones, los participantes navegaban a través de los distintos menús de juego por su cuenta.

Al término del programa de ocho semanas, a todos los participantes se les repitieron las pruebas clínicas. Para tales evaluaciones, se siguieron las instrucciones y procedimientos indicados en "Motor Control" de Shumway-Cook et al. ${ }_{(22)} \mathrm{y}$ fueron analizadas por los investigadores y parte del equipo profesional del ELEAM. En la estadística descriptiva se calculó la media, desviación estándar, mediana y rango intercuartil. Para analizar el efecto del programa, las mediciones clínicas pre y post intervención fueron comparadas usando la prueba estadística de "Wilcoxon", debido al pequeño tamaño de la muestra y la distribución no paramétrica de las variables. Para el análisis estadístico se utilizó el Software "Stata" y el nivel de significancia fue de $\mathrm{p}<0,05$. 


\section{Resultados}

De las 5 personas que terminaron el programa 3 eran de sexo femenino y 2 masculino, con edades entre 72 a 85 años $(79 \pm 4,9$ años). Las características de la muestra son presentadas en la Tabla 1. No se reportaron eventos adversos ni complicaciones.

Tabla 1. Tabla de caracterización de la muestra.

\begin{tabular}{ccccccc}
\hline Sujeto & Sexo & $\begin{array}{c}\text { Edad } \\
(\mathbf{a n n o s})\end{array}$ & $\begin{array}{c}\text { Peso } \\
(\mathbf{K g})\end{array}$ & $\begin{array}{c}\text { Talla } \\
(\mathbf{c m})\end{array}$ & Nivel educacional & $\begin{array}{c}\text { Cantidad } \\
\text { sesiones }\end{array}$ \\
\hline 1 & Masculino & 78 & 89 & 173 & Media incompleta & 24 \\
\hline 2 & Masculino & 85 & 74 & 157 & $\begin{array}{c}\text { Técnico nivel superior } \\
\text { completa }\end{array}$ & 23 \\
\hline 3 & Femenino & 82 & 47 & 155 & Media incompleta & 23 \\
\hline 4 & Femenino & 72 & 64 & 153 & Media completa & 24 \\
\hline 5 & Femenino & 78 & 64 & 150 & Básica incompleta & 24
\end{tabular}

En las mediciones clínicas antes de la intervención 2 participantes presentaron equilibrio alterado en la estación de apoyo unipodal, registrando menos de 4 segundos. No obstante, tal y como podemos observar en la Tabla 2 , la media de dicha prueba fue de $8,6 \pm 6,7$ segundos. Si consideramos que para la escala de balance de Berg un puntaje menor a 45, así como para la prueba de alcance funcional una distancia menor a $18,5 \mathrm{~cm}$ y para la SPPB un puntaje igual o menor a 6 están asociados con un mayor riesgo de caída, el comportamiento del equilibrio de los todos los participantes podría permitir clasificarlos como fuera de riesgo.

Examinando el rendimiento individual de los participantes en las pruebas clínicas post intervención podemos observar una notable mejoría, incluso en quienes registraron equilibrio alterado en la estación de apoyo unipodal. Además, según la prueba estadística de Wilcoxon todas las mediciones presentaron una diferencia significativa (SPPB $p=0,0384$, escala de balance de Berg $\mathrm{p}=0,0422$, prueba de alcance funcional $\mathrm{p}=0,0431$ y estación de apoyo unipodal $\mathrm{p}=0,0422$ ).

Tabla 2. Resultados de movilidad funcional y equilibrio.

\begin{tabular}{ccccccccccc} 
& & \multicolumn{3}{c}{ Pre intervención } & \multicolumn{5}{c}{ Post intervención } \\
\cline { 2 - 10 } & & Media & DS & Mediana & IC & Media & DS & Mediana & IC \\
\hline Movilidad & $\begin{array}{c}\text { SPPB } \\
\text { Funcional } \\
\text { (Puntos) }\end{array}$ & 8,6 & 1,1 & 9 & 2 & $10^{*}$ & 1,58 & 10 & 3 \\
\hline $\begin{array}{c}\text { Escala de } \\
\text { balance de } \\
\text { Berg } \\
\text { (Puntos) }\end{array}$ & 48,2 & 2,2 & 47 & 4 & $51,6^{*}$ & 2,3 & 52 & 4 \\
\hline $\begin{array}{c}\text { Equilibrio } \\
\text { Prueba de } \\
\text { alcance } \\
\text { funcional } \\
\text { (cm) }\end{array}$ & 29 & 6,9 & 28 & 12,9 & $33^{*}$ & 8,27 & 32 & 15,65 \\
& $\begin{array}{c}\text { Estación } \\
\text { de apoyo } \\
\text { unipodal } \\
\text { (segundos) }\end{array}$ & 8,6 & 6,7 & 6 & 12,5 & $15,4^{*}$ & 10,02 & 18 & 19,5 \\
\hline
\end{tabular}

$*=\mathrm{p}<0.05$.

\section{Discusión}

El objetivo de este estudio fue analizar el efecto de un programa de ocho semanas de ejercicios del EG KineLabs $3 D$ sobre el equilibrio y movilidad funcional en personas de 65 a 85 años autovalentes institucionalizadas. Pese a que los resultados deben ser interpretados con cautela, debido al pequeño tamaño de la muestra, las personas que participaron del programa mostraron una diferencia significativa en todas las pruebas clínicas, mejorando en promedio $14 \%$ en la SPPB, $6,6 \%$ en la escala de balance de Berg, $12,5 \%$ en la prueba de alcance funcional y $44,2 \%$ en la estación de apoyo unipodal, indicando que obtuvieron mejoras en el equilibrio y en la movilidad funcional.

En la SPPB todos los participantes mejoraron al menos por 1 punto, mientras que en la escala de balance de Berg dos mejoraron por al menos 4 puntos. Cambios que se consideran el mínimo detectable ${ }_{(29)(30)}$ y que no se deben a un error en la medición (Minimal Detectable Change, MDC). En la bibliografía revisada hasta la fecha, no existen estudios que determinen el MDC en la prueba de alcance funcional y en la estación de apoyo unipodal, así que ignoramos si los resultados son clínicamente significativos.

Si comparamos los resultados de nuestro estudio con los de otras intervenciones que utilizaron EG terapéuticos en la plataforma Kinect, encontramos que el presente trabajo tiene mayores mejoras: en la escala de balance de Berg ( 3,4 vs 3,2 vs 2,5 vs 0,66 media de puntaje) ${ }_{(31)(14)(6)}$; en la prueba de alcance funcional (4,1 vs 1,98 media en distancia) ${ }_{(13)}$; en la estación de apoyo unipodal (6,8 vs 4,44 media en tiempo) ${ }_{(13)}$.

Uno de los principales desafíos en el cuidado de las personas mayores institucionalizadas es mantener su independencia en las actividades de la vida diaria (AVD) ${ }_{(32}$, por lo que las recomendaciones actuales de actividad física en esta población están más enfocadas en conservar la capacidad funcional y mejorar la calidad de vida que en la prevención de enfermedades crónicas no transmisibles ${ }_{(32)}$.

Dentro de las recomendaciones de cuidado, está la realización de ejercicios que entrenen equilibrio. Si bien este tipo de ejercicios tiene efectos positivos en la capacidad para realizar las AVD ${ }_{(33)}$ no es concluyente respecto a que consigan disminuir de forma significante la cantidad de caídas ni el número de residentes que las sufren ${ }_{(32)}$. No obstante, Cameron et al. (34) señalan en su revisión que el entrenamiento de equilibrio con los aparatos mecánicos "Bilateral Separated Treadmill" y "Force Platform Balance Measurement and Training Device" son los únicos programas que disminuyen la cantidad de caídas en la población de las personas mayores (34). Al comparar los resultados de nuestro estudio con los de las intervenciones donde utilizaron estos aparatos, observamos mayores mejoras en la estación de apoyo unipodal (6,8 vs 1,5 media de tiempo) ${ }_{(35)}$ y en la escala de balance de Berg ( 3,4 vs 3,35 media de puntaje) ${ }_{(36)}$. 


\section{Conclusiones}

El entrenamiento con Kinect tiene, en comparación con otros sistemas de juego, beneficios especiales ya que la tecnología usada permite al usuario moverse libremente, lo que puede ofrecer mayores ventajas en tareas dinámicas ${ }_{(31)}$. Además, la retroalimentación provista por el sistema es comparable a la retroalimentación verbal en programas de ejercicio físico tradicionales, lo que podría ser suficiente para mantener a los usuarios interesados en el programa de ejercicios (31). Pese a que hay muchos aspectos positivos al usar la Kinect, también existen inconvenientes. Se requiere más espacio y los participantes necesitan estar dentro de un área calibrada, lo que hace difícil su traslado de un lugar a otro. El Software KineLabs $3 D$ también presenta algunos inconvenientes como errores inesperados del sistema o ausencia de pausa de juego, problemas que obligan a reiniciar la aplicación.

Existen limitaciones en este estudio. Primero, el pequeño tamaño de la muestra. Segundo, el tipo de estudio seleccionado es vulnerable a sesgos, en especial a los de selección y confusión. En futuras investigaciones se deberían utilizar ensayos clínicos controlados aleatorizados.

En conclusión, un programa de ocho semanas de ejercicios de Exergames en personas mayores autovalentes institucionalizadas mejoró el equilibrio y movilidad funcional. Considerando que el software es gratuito y los dispositivos de hardware son de bajo costo, KineLabs $3 D$ se presenta como una opción asequible para el entrenamiento en instituciones. Pese a que este fue un estudio piloto sus resultados son alentadores, por lo que se justifica seguir con futuras investigaciones.

\section{Referencia}

1. Van Diest M, Stegenga J, Wôrtche HJ, Verkerke GJ, Postem $\mathrm{K}$, Lamoth CJC. Exergames for unsupervised balance training at home: A pilot study in healthy older adults. Gait \& Posture. 2015; 44(1): p. 161-167.

2. Garcia J, Schoene D, Lord S, Delbaere K, Valenzuela T, Navarro, et al. A Bespoke Kinect Stepping Exergame for Improving Physical and Cognitive Function in Older People: A Pilot Study. Games for Health. 2016; 5(6): p. 382-388.

3. Debra J R. Equilibrio y Movilidad con Personas Mayores California: Paidotribo ; 2005.

4. Boucą-Machado R, Maetzler W, Ferreira JJ. What is functional mobility applied to Parkinson's disease? Journal of Parkinson's Disease. 2018; 8(1): p. 121-130.

5. Choi SD, Guo L, Kang D, Xiong S. Exergame technology and interactive interventions for elderly fall prevention: A systematic literature review. Applied Ergonomics. 2016; 1(12): p. 1-12.
6. Sato K, Kuroki K, Saiki S, Nagatomi R. Improving Walking, Muscle Strength, and Balance in the Elderly with an Exergame Using Kinect: A Randomized Controlled Trial. Games for Health Journal. 2015; 4(3): p. 161-167.

7. Hawley-Hague H, Boulton EHA, Pfeiffer K, Todd C. Older adults' perceptions of technologies aimed at falls prevention, detection or monitoring: A systematic review. International Journal of Medical Informatics. 2014; 83(6): p. 416-426.

8. Miller KJ, Adair BS, Pearce AJ, Said CM, Ozanne E, Morris MM. Effectiveness and feasibility of virtual reality and gaming system use at home by older adults for enabling physical activity to improve health-related domains: A systematic review. Age and Ageing. 2014; 43(2): p. 188-195.

9. Donath L, Rössler R, Faude O. Effects of Virtual Reality Training (Exergaming) Compared to Alternative Exercise Training and Passive Control on Standing Balance and Functional Mobility in Healthy Community-Dwelling Seniors: A Meta-Analytical Review. Sports Medicine. 2016; 46(9): p. 1293-1309.

10. Skjæret N, Nawaz A, Morat T, Schoene D, Helbostad J, Vereijken B, et al. Exercise and rehabilitation delivered through exergames in older adults: An integrative review of technologies, safety and efficacy. International Journal of Medical Informatics. 2015; 85(1): p. 1-16.

11. Konstantinidis EI, Bamparopoulos G, Bamidis PD. Moving Real Exergaming Engines on the Web: The webFitForAll Case Study in an Active and Healthy Ageing Living Lab Environment. IEEE Journal of Biomedical and Health Informatics. 2017; 21(3): p. 859-866.

12. Marston HR, Woodbury A, Gschwind YJ, Kroll M, Fink D, Eichberg S, et al. The design of a purpose-built exergame for fall prediction and prevention for older people. European Review of Aging and Physical Activity. 2015; 12(1): p. 1-13.

13. Kayama H, Okamoto K, Nishiguchi S, Yukutake T, Tanigawa T, Nagai K, et al. Efficacy of an Exercise Game Based on Kinect in Improving Physical Performances of Fall Risk Factors in Community-Dwelling Older Adults. Games for Health. 2013; 2(4): p. 247-252.

14. Hsieh WM, Chen CC, Wang SC, Tan SY, Hwang YS, Chen $\mathrm{SC}$, et al. Virtual reality system based on Kinect for the elderly in fall prevention. Technology and Health Care. 2014; 22(1): p. 27-36.

15. Chen CC. Improvement in the physiological function and standing stability based on kinect multimedia for older people. Journal of Physical Therapy Science. 2016; 28(4): p. 13431348.

16. Bonnechère B, Jansen B, Omelina L, Van S, Jan S. The use of commercial video games in rehabilitation: a systematic review. International Journal of Rehabilitation Research. 2016; 39(4): p. 277-290. 
17. Organizacion Mundial de la Salud. Recomendaciones mundiales sobre actividad física para la salud ; 2010.

18. Tong RKY, Hang CH, Chong LKW, Lam NKF. KineLabs 3D motion software platform using Kinect Elderly Exercise and Stroke Rehabilitation. Computerized Healthcare (ICCH), 2012 International Conference on. 2012; 1(1): p. 164-165.

19. Manterola C, Otzen T. Estudios Experimentales $2^{\text {a }}$ Parte. Estudios Cuasi-Experimentales. International Journal of Morphology. 2015; 33(1): p. 382-387.

20. Miyamoto ST, Lombardi I, Berg KO, Ramos LR, Natour J. Brazilian version of the Berg balance scale. Brazilian Journal of Medical and Biological Research. 2004; 37(9): p. 14111421.

21. Bogle L, Newton R. Use of the Berg Balance Test to Predict Falls in Elderly Persons. Physical Therapy. 1996; 76(6): p. 576-583.

22. Duncan P, Weiner D, Chandler J, Studenski S. Functional Reach: A New Clinical Measure of Balance. Journal of Gerontology. 1990; 45(6): p. 192-197.

23. Shumway-Cook A, Woollacott M. Motor Control: Theory and practical applications. Baltimore: Lippincott Williams \& Wilkins; 2012.

24. Thomas JI, Lane JV. A pilot study to explore the predictive validity of 4 measures of falls risk in frail elderly patients. Archives of Physical Medicine and Rehabilitation. 2005; 86(8): p. 1636-1640.

25. Roberts H. Normative Values for the Unipedal Stance Test with Eyes Open and Closed Normative Values for the Unipedal Stance Test with Eyes Open and Closed. Journal of Geriatric Physical Therapy. 2016; 30(1): p. 9-15.

26. Ministerio de Salud. Manual de Prevención de Caídas en el Adulto Mayor Santiago: Duplika Ltda.; 2010.

27. Treacy D, Hassett L. The Short Physical Performance Battery. Journal of Physiotherapy. 2018; 64(1): p. 61.

28. Veronese N, Bolzetta F, Toffanello D, Zambon S, De Rui M, Perissinotto E, et al. Association Between Short Physical Performance Battery and Falls in Older People: The Progetto Veneto Anziani Study. Rejuvenation Research. 2014; 17(3): p. 278-283.

29. Pirovano M, Surer E, Mainetti R, Lanzi PL, Alberto Borghese N. Exergaming and rehabilitation: A methodology for the design of effective and safe therapeutic exergames. Entertainment Computing. 2016; 14(1): p. 55-65.

30. Perera S, Mody SH, Woodman RC, Studenski SA. Meaningful change and responsiveness in common physical performance measures in older adults. Journal of the American Geriatrics Society. 2006; 54(5): p. 743-749.
31. Donoghue D, Murphy A, Jennings A, McAuliffe A, O'Neil $\mathrm{S}$, Charthaigh EN, et al. How much change is true change? The minimum detectable change of the Berg Balance Scale in elderly people. Journal of Rehabilitation Medicine. 2009; 41(5): p. 343-346.

32. Bieryla KA, Dold NM. Feasibility of Wii Fit training to improve clinical measures of balance in older adults. Clinical Interventions in Aging. 2013; 8(6): p. 775-781.

33. de Souto Barreto P, Morley JE, Chodzko-Zajko W, H. Pitkala K, Weening-Djiksterhuis hE, Rodriguez-Mañas L, et al. Recommendations on Physical Activity and Exercise for Older Adults Living in Long-Term Care Facilities: A Taskforce Report. Journal of the American Medical Directors Association. 2016; 17(5): p. 381-392.

34. Crocker T, Forster A, Young J, Brown L, Ozer S, Smith $\mathrm{J}$, et al. Physical rehabilitation for older people in long-term care. Cochrane Database of Systematic Reviews. 2013; 1(2): p. 1-269.

35. Cameron ID, Dyer SM, Panagoda CE, Murray GR, Hill $\mathrm{KD}$, Cumming RG, et al. Interventions for preventing falls in older people in care facilities and hospitals. Cochrane Database of Systematic Reviews. 2012; 9(12): p. 1-181.

36. Shimada H, Obuchi S, Furuna T, Suzuki T. New intervention program for preventing falls among frail elderly people: The effects of perturbed walking exercise using a bilateral separated treadmill. American Journal of Physical Medicine and Rehabilitation. 2004; 83(7): p. 493-499.

37. Sihvonen SE, Sipilä S, Era PA. Changes in Postural Balance in Frail Elderly Women during a 4-Week Visual Feedback Training : A Randomized Controlled Trial. Gerontology. 2004; 50(1): p. 87-95.

38. Organización Mundial de la Salud. Global report on falls prevention in older age Geneva; 2007.

39. Sihvonen S, Sipilä S, Taskinen S, Era P. Fall incidence in frail older women after individualized visual feedback-based balance training. Gerontology. 2004;: p. 411-416.

40. Bainbridge E, Bevans S, Keeley B, Oriel K. The effects of the Nintendo Wii Fit on community-dwelling older adults with perceived balance deficits: A pilot study. Physical and Occupational Therapy in Geriatrics. 2011; 29(2): p. 126-135 